\title{
Data Analysis and Classification of mHealth Shimmer2 Sensor Data sets for Human Physical Activities Recognition
}

\author{
Maria Navin J R, Sridevi N, Pankaja R
}

\begin{abstract}
Human physical activity recognition is one of the important attributes for well being of human health and activity recogni-tion field. A physical signal with respect to time based directed toward recognizes human activity events is projected, in the view of this work usage wearable accelerometer sens-ing devices were implanted taking place the human body lo-cation on the upper body Chest Sensor (CS), Left Ankle Sen-sor (LAS) and Right Lower Arms Sensor (RLAS). Accelerome-ter feature extracted based acceleration signals with respect to time, physical appearance of the accelerometer $x, y$, and $z$ dimension values reported/recorded using shimmer2 weara-ble sensor device is recommended at the categorization of the 10 different users was performed 12 different types human activities, including vigorous and moderate activities. User ages between 24 to 29 years and human body weight $(\mathrm{HBW})$ are 53 to $83 \mathrm{Kg}=\mathrm{m} 2$. Results were on view a large validity per-formance precision and recall were getting 95 for each human activities. The whole classifiers accuracy results for all combi-nation of the feature set of all sensors is $98 \%$. The considered work could be used to observe the human body motion of different body location of users and to perform data analysis and classification.
\end{abstract}

Keywords - Data Analysis, Activity Recognition, Wearable Sensor, Physical Activity, Classification.

\section{INTRODUCTION}

Monitoring human Daily Physical Activity (DPA) and ascertaining human body orientation/motion is useful in appropriate ways; the advantages of Physical action reach out a long ways past weight administration. Research indicates that humans involved in physical activities can prevent the hazard and future illnesses. The physical movements can also enhance personnel health conditions. Consistent physical movement can protect humans from various medical issues including the improvement of individual weight control designs and the gradual recovery of patients. Physical activity considered for analysis are 12 which includes stair climbing,

Revised Manuscript Received on July 22, 2019.

Maria Navin J R, Dept. of ISE, Sri Venkateshwara College of Engineering, Bangalore, Karnataka, INDIA.

Sridevi N, Dept. of CSE, Sri Venkateshwara College of Engineering, Bangalore, Karnataka, INDIA.

Pankaja R, Dept. of ISE, Sri Venkateshwara College of Engineering, Bangalore, Karnataka, INDIA.

Sri Venkateshwara College of Engineering, Bangalore, Karnataka, INDIA.

Email_marianavin.jr@gmail.com, n.sridevi5@gmail.com, pankaja.ssu@gmail.com standing upright, sitting, laying down, walking, bend forward, elevating the arms front, bending the knees, cycling, jogging, running and jumping etc. Accordingly, the creators urge the group to add to this inventive stage by supporting the utilization of the most recent sensors, consolidating new behavioural calculations or basically making utilization of it for the improvement of versatile wellbeing mobile applications [1]. In a smart world (the physical environment where various sensors embedded in our daily routines to monitored distinctive parts of our living), low-cost wireless sensors can observe and monitor the characteristics and state of action of individuals. Different set of populations including diseased patients and healthy individuals can be monitored successfully using the acceleration based activity. Recently obesity has been a critical issue among population because of physical latency so analysis of human Activities is a concern [2].

\section{LITERATURE SURVEY}

Human Physical Activity Recognition (HPAR) using wearable sensors is a very widespread research subject. Using mobile phone motion accelerometer sensor data to perform Human Activity Recognition (HAR), an undertaking which includes distinguishing the physical movement a client is performing. To actualize this framework creator gathered named accelerometer sensor data information from twenty-nine subject as they accomplished everyday activities such as walking, jogging, climbing stairs, sitting, and standing, and then aggregated this time series data into examples that encapsulate the user activity over 10 -second timeout in each activity. Creators at that point utilized the subsequent preparing information to instigate a prescient model for movement acknowledgment. Earlier work by Kwapisz J R et al[3]. The whole process for activity recognition beings with gathering the raw data, specifically, motion data. The inertial sensor is an adequate solution to detect motion of the subject. This inertial sensor replies to stimulants by generating signals that can be analyzed and understood. Monitoring physical activity by using wearable sensors reviews of various classification methods used to recognize Daily Human Activities (DHA). Three inertial sensor units were used in the healthy subject at key points of the chest, right thigh and left ankle [4][5]. An absence of sufficient physical movement is a huge issue in our general public in light of the fact that physical inertia drastically

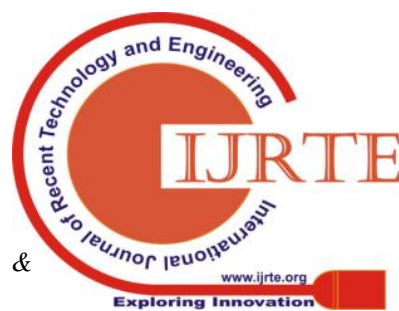


expands the wellbeing dangers for some, maladies, including cardiovascular sickness.

\subsection{Feature Generation}

256 readings were generated from accelerometer using which informative features were obtained. Each of those readings had three dimensional values with respect to the axes $\mathrm{x}, \mathrm{y}$ and z. We brought about a twenty one features in each position like at the position Chest Sensor (CS), Left ankle Sensor (LAS) and Right Lower Arm Sensor (RLAS), in spite of the fact that these are altogether variations of only seven essential basic features. Human activity recognition from shimmer2 sensor data sets is normally gone before by a highlights extraction step. Signal qualities, for example, time-area and recurrence space highlights are broadly utilized for feature calculation. In this proposed work the author have used time domain features. Time-domain features include Mean Absolute Value (MAV), Harmonic Mean (HM), Variance (VR), Root Mean Square (RMS), Skewness (SK), Kurtosis (KT) and Simple Squared Integral (SSI) [6].

\section{METHODOLOGY}

Using shimmer2 wearable sensor device primary mHealth data was generated by Banos, et.al[1]. This work mHealth data is used as secondary data for human activity recognition [7][8]. After we have selected the mHealth data sets for Activity Recognition (AR) we can setup new Human Activity Recognition (HAR) system which can divide $70 \%$ of train data and $30 \%$ of test data for extracting features. To get mHealth data then divide data into $5 / \mathrm{sec}$ segment then generate time domain based feature that we are based 256 readings of acceleration sensor data readings, each reading have $\mathrm{x}, \mathrm{y}$ and co-ordinates equivalent to the three-dimension values[9].

Table 1. Number of instances per activity

\begin{tabular}{|c|c|}
\hline $\begin{array}{c}\text { Physical Activity } \\
\text { Number of Instances }\end{array}$ & $\begin{array}{c}\text { Number of } \\
\text { Instances }\end{array}$ \\
\hline A1: Climbing Stairs & 1000 \\
\hline A2: Cycling & 1000 \\
\hline A3: Front elevation of Arms & 1000 \\
\hline A4: Jogging & 1000 \\
\hline A5: Jump front and back & 1000 \\
\hline A6: Knee Bending & 1000 \\
\hline A7: Laying Down & 1000 \\
\hline A8: Running & 1000 \\
\hline A9: Sitting and Relaxing & 1000 \\
\hline A10: Standing Still & 1000 \\
\hline A11: Waist bends forward & 1000 \\
\hline A12: Walking & 1000 \\
\hline Total & 12000 \\
\hline
\end{tabular}

MHealth data contains 10 persons of varying age, height, weight and different gender and each user was done 12 different types of activity that are shown in table 2 . The feature was extracted descending window wt of $5 / \mathrm{sec}$ time duration Shimmer2 wearable data in $\mathrm{X}, \mathrm{Y}$ and $\mathrm{Z}$ dimensions of the three important body position and the important features are extracted and derived from every 256 instances in each physical activity this window to recognize the movement as being one of twelve conceivable alternatives. The sampler frequency was recorded the accelerometer data at $50 \mathrm{~Hz}$ capacity. A connection of one $1 / \mathrm{sec}$ is considered for sliding the window on the shimmer 2 sensor data. Time domain features are intended on the duration of $5 / \mathrm{sec}$ and four features sets (TDFS1, TDFS2, TDFS3 and TDF4) of 12000 instances are obtained in each activity from each placed of accelerometer sensors, placed on the Chest, Right Lower Arms (RLA) and Left Ankle(LA), respectively. Table 1 shows the dispersion of instances of the different daily physical activities in the feature sets. Time domain features are calculated for all twelve different types of physical activities. The aggregate removed list of capabilities is haphazardly disconnected into two sets. The main preparing dataset contains $70 \%$ of the information cases from the time area highlights set and this $70 \%$ dataset utilized for preparing the classifiers, though the rest of the informational indexes considered as a testing set of the information occurrences from the time-space include set and are utilized for testing the characterization precision introduction of the classifiers. The information exactness consequences of every one of the twelve of the physical exercises utilizing tri-quickening agent wearable sensors and a blend of each of the four sensors body area are expressed in the following allotments.

\section{RESULTS AND DISCUSSIONS}

Human Physical Activity Recognition (HPAR) the machine learning algorithms using accelerometer sensor data can be balanced in human physical activity recognition in different human body aspects and activities based on Precision, Recall, and F-measure. The accuracy or positive prescient esteem (PPV) is characterized as the extent of occasions that has a place with a class by the aggregate occurrences, including TP and FP ordered by the classifier as be proper to this element class.

F-measure is retrieval data evidence. It contemplates both exactness and review and as a rule joins them into a weighted consonant mean. It ponders both exactness and review and as a rule joins them into a weighted consonant mean, where it achieves its best an incentive at 1 and the most exceedingly terrible incentive at 0 .

As a general measure of precision, F1 has been broadly utilized as a part of prior works for the appraisal of investigation execution. We utilize F-measures to enable us to appraise how exact and quickly we can make a proposition for the proper articles.

Table 2. Results of Naive Bayes Classifier with different sensor positions

\begin{tabular}{|c|c|c|c|c|}
\hline \multirow{2}{*}{$\begin{array}{c}\text { Feature } \\
\text { Set }\end{array}$} & \multirow{2}{*}{$\begin{array}{l}\text { Sensor } \\
\text { Position }\end{array}$} & \multicolumn{3}{|c|}{ Naive Bayes } \\
\hline & & Precision & Recall & F1 \\
\hline TDFS1 & RLAS & 0.93 & 0.92 & 0.92 \\
\hline TDFS2 & CHEST & 0.89 & 0.91 & 0.91 \\
\hline TDFS3 & LAS & 0.92 & 0.90 & 0.92 \\
\hline TDFS4 & ALL & 0.92 & & 0.93 \\
\hline
\end{tabular}


Table 3. Results of Support Vector Machine Classifier with different sensor positions

\begin{tabular}{|c|c|c|c|c|}
\hline Feature & \multirow{2}{*}{$\begin{array}{c}\text { Sensor } \\
\text { Set }\end{array}$} & Position & \multicolumn{3}{|c|}{ Support Vector Machine } \\
\cline { 3 - 5 } & & Precision & Recall & F11 \\
\hline TDFS1 & RLAS & 0.94 & 0.95 & 0.91 \\
\hline TDFS2 & CHEST & 0.93 & 0.95 & 0.90 \\
\hline TDFS3 & LAS & 0.94 & 0.95 & 0.91 \\
\hline TDFS4 & ALL & 0.94 & 0.95 & 0.92 \\
\hline
\end{tabular}

Table 4. Results of Convolution Neural Networks Classifier with different sensor positions

\begin{tabular}{|c|c|c|c|c|}
\hline \multirow{2}{*}{$\begin{array}{c}\text { Feature } \\
\text { Set }\end{array}$} & \multirow{2}{*}{$\begin{array}{c}\text { Sensor } \\
\text { Position }\end{array}$} & \multicolumn{3}{|c|}{$\begin{array}{c}\text { Convolution Neural } \\
\text { Networks }\end{array}$} \\
\cline { 3 - 5 } & & Precision & Recall & F1 \\
\hline TDFS1 & RLAS & 0.96 & 0.97 & 0.97 \\
\hline TDFS2 & CHEST & 0.97 & 0.95 & 0.96 \\
\hline TDFS3 & LAS & 0.98 & 0.97 & 0.97 \\
\hline TDFS4 & ALL & 0.97 & 0.97 & 0.97 \\
\hline
\end{tabular}

Table 5. Classification accuracy of RLAS/TDFS1 activities

\begin{tabular}{|c|c|c|c|}
\hline \multirow{2}{*}{ Activity } & \multicolumn{3}{|c|}{ RLAS/TDFS1 } \\
\cline { 2 - 4 } & NB & SVM & CNN \\
\hline A1 & 91.24 & 94.23 & 96.12 \\
\hline A2 & 92.34 & 93.98 & 96.89 \\
\hline A3 & 93.89 & 94.90 & 97.90 \\
\hline A4 & 93.80 & 95.11 & 98.12 \\
\hline A5 & 92.24 & 93.90 & 97.45 \\
\hline A6 & 93.04 & 94.58 & 97.65 \\
\hline A7 & 94.14 & 94.29 & 97.03 \\
\hline A8 & 92.27 & 93.23 & 97.13 \\
\hline A9 & 92.55 & 93.45 & 97.40 \\
\hline A10 & 91.75 & 92.98 & 97.23 \\
\hline A11 & 92.46 & 92.79 & 96.45 \\
\hline A12 & 93.16 & 95.04 & 96.07 \\
\hline Average & 92.74 & 94.04 & 97.12 \\
\hline
\end{tabular}

Table 6. Classification accuracy of CHEST/TDFS2 activities

\begin{tabular}{|c|c|c|c|}
\hline \multirow{2}{*}{ Activity } & \multicolumn{3}{|c|}{ CHEST/ TDFS2 } \\
\cline { 2 - 4 } & NB & SVM & CNN \\
\hline A1 & 91.23 & 94.56 & 98.89 \\
\hline A2 & 91.34 & 94.09 & 97.12 \\
\hline A3 & 91.2 & 94.35 & 98.14 \\
\hline A4 & 91.04 & 95.2 & 97.84 \\
\hline A5 & 91.2 & 94.21 & 97.35 \\
\hline A6 & 91.34 & 94.77 & 97.19 \\
\hline A7 & 91.01 & 95.41 & 98.04 \\
\hline A8 & 91.8 & 94.39 & 98.57 \\
\hline A9 & 91.12 & 94.28 & 97.29 \\
\hline A10 & 91.57 & 93.79 & 97.45 \\
\hline A11 & 91.35 & 95.62 & 97.94 \\
\hline A12 & 91.16 & 94.05 & 97.3 \\
\hline Average & 91.28 & 94.56 & 97.76 \\
\hline \multicolumn{3}{|c}{} \\
\hline
\end{tabular}

\subsection{Acceleration Sensor Device Located on Different Body}

The accuracy of machine learning algorithms the Arm Sensor (RLAS), Chest Sensor (CS) and Left Ankle TDFS3, and TDFS4 are contained within of 21 extracted features from the three dimensional acceleration sensor data in three $\mathrm{x}, \mathrm{y}$ and $\mathrm{z}$ dimensions. The accuracy results of three time domain feature testing set to the obtained classification accuracy of three classifiers results in Table 2, 3 and 4. 
Table 7. Classification accuracy of LAS/ TDFS3 activities

\begin{tabular}{|c|c|c|c|}
\hline \multirow{2}{*}{ Activity } & \multicolumn{3}{|c|}{ LAS/ TDFS3 } \\
\cline { 2 - 4 } & NB & SVM & CNN \\
\hline A1 & 91.24 & 94.23 & 98.12 \\
\hline A2 & 92.34 & 93.98 & 98.89 \\
\hline A3 & 91.89 & 94.9 & 98.9 \\
\hline A4 & 93.8 & 95.11 & 98.12 \\
\hline A5 & 92.24 & 95.9 & 97.45 \\
\hline A6 & 91.04 & 94.58 & 97.65 \\
\hline A7 & 94.14 & 94.29 & 97.03 \\
\hline A8 & 92.27 & 93.23 & 98.13 \\
\hline A9 & 91.55 & 93.45 & 98.4 \\
\hline A10 & 91.75 & 94.98 & 98.23 \\
\hline A11 & 91.46 & 92.79 & 98.45 \\
\hline A12 & 91.84 & 93.44 & 98.07 \\
\hline Average & 92.13 & 94.24 & 98.12 \\
\hline
\end{tabular}

Table 8. Classification accuracy of ALL/ TDFS4 activities

\begin{tabular}{|c|c|c|c|}
\hline \multirow{2}{*}{ Activity } & \multicolumn{3}{|c|}{ ALL/ TDFS4 } \\
\cline { 2 - 4 } & NB & SVM & CNN \\
\hline A1 & 92.51 & 94.59 & 98.7 \\
\hline A2 & 93.9 & 94.01 & 99.13 \\
\hline A3 & 92.48 & 95.26 & 99.24 \\
\hline A4 & 92.04 & 94.37 & 98.89 \\
\hline A5 & 93.15 & 94.98 & 98.17 \\
\hline A6 & 93.69 & 94.66 & 98.22 \\
\hline A7 & 92.48 & 94.7 & 98.61 \\
\hline A8 & 92.61 & 94.13 & 99.04 \\
\hline A9 & 93.73 & 94.07 & 98.26 \\
\hline A10 & 92.39 & 94.19 & 98.33 \\
\hline A11 & 93.18 & 94.79 & 98.19 \\
\hline A12 & 93.6 & 94.97 & 98.54 \\
\hline Average & 92.98 & 94.56 & 98.61 \\
\hline
\end{tabular}

accuracy values for the sitting and relaxing and standing still in different positions of accelerometer data.

Precision, recall, and F-measure are stated for all four different sensor positions. The overall results of Precision, Recall and F1 measures for Naive Bayes (NB) are 0.92, 0.91, 0.93 and support vector machine (SVM) are 0.94, 0.95 and 0.92 and finally, Convolution Neural Networks algorithms are getting more values $0.97,0.97$ and 0.97 respectively. The NB classifier performed very poorly. Classification results of SVM classifiers are comparatively better than NB classifier. The CNN algorithm was comparatively getting more precision, recall and f-measure values for all physical activities is almost greater than are equal to 0.97 . The best precision, recall and f-measure results are fulfilled by the CNN algorithm.

Human physical activities (HPA) comprise the body movement of various body areas, contingent upon the sort of physical movement. For instance, the exercises of stopping and running elaborate more movement of the entire body. Hence, different human physical activities classify accurately by synthesis of the frequency and time area appearance derived from the different human body location. In the earlier segments, physical activities had low
Furthermore, sometimes cycling and walking are below par classified by the shimmer2 accelerometer data positioned on the Right Lower Arms (RLA) and Chest. Sitting and relaxing and standing still activities have deep accuracy values, when a shimmer2 accelerometer device was located at the position of the Left Ankle Sensor (LAS). Therefore, in this portion, time domain features extracted from all three locations of sensors are connected and all three classifiers are functional to differentiate the set of human different body motion. Time domain features set for all three important position [TDFS4 = TDFS1 TDFS2 TDFS3]. The combined all RLMS, CHEST, LAS all three sensors classification and accuracy results are summarized in Table 5, 6,7 and 8. The overall classification accuracy of all three sensors position combined is observed to be $98 \%$ with overall Precision, Recall and F-measure values are $0.96,0.97$ and 0.95 respectively. 


\section{CONCLUSION}

The shimmer2 wearable sensors devices are located on different human body locations namely Chest, Right Lower Arms Sensor (RLAS) and Left Ankle Sensor (LAS) respectively, to recognize 12 various types of Human Physical Activity (HPA). Features were isolated based on time domain from the shimmer2 acceleration data and the appearance of three different advanced machine learning classifiers are evaluated for every proposed position. The CNN algorithms are found to be the accuracy of the very good classifier between among the three advanced classifiers methods in all location. A normal classification exactness of $98 \%$ is achieved by $\mathrm{CNN}$ algorithms. Moreover, the collective all three acceleration Time Domain Feature Set (TDFS4) is found to be $92 \%, 94 \%$, and $97 \%$ for the NB, SVM and CNN algorithms respectively. Our proposed methods for data analysis and classification of shimmer2 wearable accelerometer sensor data was useful for identifying the healthy lifestyle for people and also survey the physical movement of individuals and the patient.

\section{REFERENCES}

[1] Banos, O., Garcia, R., Holgado-Terriza, J.A., Damas, M., Pomares, H., Rojas, I., Saez, A., Villalonga, C.: mhealthdroid: a novel framework for agile development of mobile health applications. In: International Workshop on Ambient AssistedLiving, Springer (2014) 91-98.

[2] McAdams, E.T., Gehin, C., Noury, N., Ramon, C., Nocua, R., Massot, B., Oliveira, A., Dittmar, A., Nugent, C.D., Laughlin, J.: Biomedical sensors for ambient assisted living. In: Advances in Biomedical Sensing, Measurements, Instrumentation and Systems. Springer (2010) $240\{262$.

[3] Kwapisz, J.R., Weiss, G.M., Moore, S.A.: Activity recognition using cell phone accelerometers. ACM SigKDD Explorations Newsletter 12(2) (2011) 74-82.

[4] Cleland, I., Kikhia, B., Nugent, C., Boytsov, A., Hallberg, J., Synnes, K., McClean, S., Finlay, D.: Optimal placement of accelerometers for the detection of everyday activities. Sensors 13(7) (2013) 9183-9200.

[5] Yogesh, K., et al.: Instance based human physical activity (hpa) recognition using shimmer 2 wearable sensor data sets. In: Advances in Computing, Communications and Informatics (ICACCI), 2017 International Conference on, IEEE (2017) 995-999.

[6] Arif, M., Kattan, A., Physical activities monitoring using wearable acceleration sensors attached to the body. PloS one 10(7) (2015) e0130851.

[7] Eduardo Casilari , José-Antonio Santoyo-Ramón and José-Manuel Cano-García., Analysis of Public Datasets for Wearable Fall Detection Systems Sensors 2017, 17, 1513; doi:10. 3390/s17071513.

[8] Daniela Micucci, Marco Mobilio and Paolo Napoleno,. UniMiB SHAR: A Dataset for Human Activity Recognition Using Acceleration Data from Smart phones Appl. Sci. 2017, 7, 1101; doi:10.3390/app 7101101.

[9] Doreswamy, Yog sh K., Group Based Human Physical Activity Classification (Gbhac) Using Shimmer2 Wearable Sensor Data Sets IJLTET, Special Issue SACAIM 2017, pp. 006-012. 